Research article

\title{
Changes in expression of Kv7.5 and Kv7.2 channels in dorsal root ganglion neurons in the streptozotocin rat model of painful diabetic neuropathy
}

\author{
Laiche Djouhri $^{\mathrm{a}, *}$, Asad Zeidan ${ }^{\mathrm{a}}$, Seham A. Abd El-Aleem ${ }^{\mathrm{b}, \mathrm{c}}$

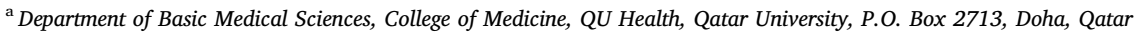 \\ ${ }^{\mathrm{b}}$ University of Manchester, Manchester UK \\ ${ }^{\mathrm{c}}$ Department of Histology and Cell Biology, Minia Faculty of Medicine, Minia University, Minia, Egypt
}

\section{A R T I C L E I N F O}

\section{Keywords:}

Diabetic neuropathy

Primary sensory neurons

$\mathrm{K}^{+}$channels

\begin{abstract}
A B S T R A C T
Diabetic peripheral neuropathic pain (DPNP), the most debilitating complication of diabetes mellitus, is resistant to current therapy. The pathogenesis of DPNP is still elusive, but several mechanisms have been proposed including abnormal hyperexcitability of dorsal root ganglion (DRG) neurons. The underlying molecular mechanisms of such aberrant hyperexcitability are incompletely understood. Using the streptozotocin (STZ) rat model of DPNP, we have recently provided evidence implicating neuronal $\mathrm{K}_{\mathrm{v}} 7$ channels that normally exert a powerful stabilizing influence on neuronal excitability, in the abnormal hyperexcitability of DRG neurons and in pain hypersensitivity associated with DPNP. In the present immunohistochemical study, we sought to determine whether $\mathrm{K}_{\mathrm{v}} 7.2$ and/or $\mathrm{K}_{\mathrm{v}} 7.5$ channel expression is altered in DRG neurons in STZ rats. We found 35 days postSTZ: (1) a significant decrease in $\mathrm{K}_{\mathrm{v}} 7.5$-immunoreactivity in small $(<30 \mu \mathrm{m})$ DRG neurons (both IB4 positive and IB4 negative) and medium-sized $(30-40 \mu \mathrm{m})$ neurons, and (2) a significant increase in $\mathrm{K}_{\mathrm{v}} 7.2$-immunoreactivity in small $(<30 \mu \mathrm{m})$ neurons, and a non-significant increase in medium/large neurons. The decrease in $\mathrm{K}_{\mathrm{v}} 7.5$ channel expression in small and medium-sized DRG neurons in STZ rats is likely to contribute to the mechanisms of hyperexcitability of these neurons and thereby to the resulting pain hypersensitivity associated with DPNP. The upregulation of $\mathrm{K}_{\mathrm{v}} 7.2$ subunit in small DRG neurons may be an activity dependent compensatory mechanism to limit STZ-induced hyperexcitability of DRG neurons and the associated pain hypersensitivity. The findings support the notion that $\mathrm{K}_{\mathrm{v}} 7$ channels may represent a novel target for DPNP treatment.
\end{abstract}

\section{Introduction}

Diabetic peripheral neuropathy (DPN) is the most debilitating complication of diabetes mellitus (e.g. [1]) and the most common cause of peripheral neuropathic pain (PNP). Indeed up to $50 \%$ of people with DPN have some degree of PNP [1-3]. Diabetic PNP (DPNP) is characterized in humans by unpleasant sensations (dysesthesias/paresthesia) as well as spontaneous pain and pain hypersensitivity to mechanical and thermal stimuli [4]. These symptoms are often concurrent with loss of sensation in the feet that commonly results in foot ulcers that may progress to limb amputations [5,6]. DPNP is resistant to currently available drugs that either have limited efficacy and/or significant adverse side effects. The pathogenesis of DPNP is still illusive, but several mechanisms have been proposed including hyperexcitability of dorsal root ganglion (DRG) neurons (see [7]). Indeed several studies using animal models of PNP including the streptozotocin (STZ) model, have shown that both C- and A-fiber DRG neurons exhibit abnormal spontaneous activity (SA), the key characteristic of neuronal hyperexcitability [8-11]. Such SA is believed to drive the changes in the CNS and to induce "central sensitization", an important mechanism of PNP. The underlying molecular mechanisms of the abnormal SA and hyperexcitability of DRG neurons associated with DPNP remain largely unknown, but could result from a decrease in expression of voltagegated $\mathrm{K}_{\mathrm{v}} 7$ potassium channels that are tonically active near the resting membrane potential. This is because these channels mediate an outward $\mathrm{M}$ current that normally exerts a powerful stabilizing effect on neuronal excitability (see $[12,13]$ ).

$\mathrm{K}_{\mathrm{v}} 7$ (KCNQ) $\mathrm{K}^{+}$channels are composed of five members: the cardiac channel $\mathrm{K}_{\mathrm{v}} 7.1$, and the neuronal $\mathrm{K}_{\mathrm{v}} 7.2-7.5$ channels which underlie the neuronal $\mathrm{M}$ current that acts as a "dynamic brake" on repetitive action potential discharges (see [14]). Therefore, decreased expression of $\mathrm{K}_{\mathrm{v}} 7 /$ $\mathrm{M}$ channels could result in membrane depolarization, and render

\footnotetext{
* Corresponding author.

E-mail address: 1djouhri@qu.edu.qa (L. Djouhri).
} 
neurons more prone to action potential firing [12]. Homo-or heteromultimer assemblies of $\mathrm{K}_{\mathrm{v}} 7.2-7.5$, primarily $\mathrm{K}_{\mathrm{v}} 7.2 / 7.3$, underlie the $\mathrm{M}$ current in neurons (see [13]). $\mathrm{K}_{\mathrm{v}} 7.2,7.3$ and 7.5 channels are expressed at all levels of the pain pathway, including somata of DRG neurons and their axons, peripheral and central terminals (see [15]). $\mathrm{K}_{\mathrm{v}} 7.2$ was shown to be the predominant subunit in C-type DRG neurons [16], but another study [17] reported that $\mathrm{K}_{\mathrm{v}} 7.5$ is the primary subunit expressed in these neurons. Furthermore, $\mathrm{K}_{\mathrm{v}} 7.2$ and $\mathrm{K}_{\mathrm{v}} 7.3$ subunits were found to be down-regulated in nociceptive DRG neurons after nerve injury, and in an animal model of bone cancer $[16,18,19]$. However, other studies reported no change (or even a transient increase) in the expression of the $\mathrm{K}_{\mathrm{v}} 7.2, \mathrm{~K}_{\mathrm{v}} 7.3$ or $\mathrm{K}_{\mathrm{v}} 7.5$ channel proteins in DRG neurons following spinal nerve injury (see [15]. As far as we know, only one recent study examined expression of these subunits in DRG neurons in the rat STZ model of DPNP and found significant decreases in $\mathrm{K}_{\mathrm{v}} 7.2$, Kv7.3 and Kv7.5 at both mRNA and protein levels [20]. Thus, further studies are required to unravel the apparent discrepancies between the aforementioned studies.

Using the STZ model of DPNP, in vivo electrophysiology and behavioral assays, we have recently provided evidence implicating $\mathrm{K}_{\mathrm{v}} 7$ channels in hyperexcitability of DRG neurons [21] and the associated pain hypersensitivity [22]. In the present immunohistochemical study, we sought to examine whether expression of $\mathrm{K}_{\mathrm{v}} 7.2$ and/or $\mathrm{K}_{\mathrm{v}} 7.5$ channels is altered in DRG neurons in STZ rats. We focused on these subunits because they are clearly expressed in rodent DRG neurons, and because we do not have reliable and selective antibodies for the other neuronal Kv7.4 and Kv7.3 subunits. We found, 5 weeks post-STZ, a significant decrease in $\mathrm{K}_{\mathrm{v}} 7.5$-immunoreactivity in small $(<30 \mu \mathrm{m})$ and medium $(30-40 \mu \mathrm{m})$ DRG neurons, and a significant increase in $\mathrm{K}_{\mathrm{v}} 7.2$ immunoreactivity in small $(<30 \mu \mathrm{m})$ DRG neurons.

\section{Materials and methods}

\subsection{Experimental animals}

Male Sprague Dawley rats (200-280 g, Charles River, UK) were used in the present study. They were housed in a room in the animal house of Liverpool University at room temperature between 20 and $26^{\circ} \mathrm{C}$ while under a 12 -h (h) dark and light cycle. They had free access to food and water. The experimental procedures were approved by the University of Liverpool Ethical review committee, and complied throughout with the 1986 UK Scientific Procedures Animals Act. Based on previously established criteria of exploratory activity and weight gain [23], all rats appeared healthy throughout the experiments. However, as we reported previously [22], the body weights of STZ-diabetic rats were significantly lower than controls throughout the study duration (see also Results).

\subsection{Animal model of PNP}

We used the STZ (streptozotocin) rat model of DPNP that involves a single injection of STZ $(60 \mathrm{mg} / \mathrm{kg}$, i.p.) after an overnight fast as reported previously (see [22]). We used this model because: (1) it is more commonly used than other models that have been developed to investigate the pathophysiology of DPNP, and (2) It has been shown by various studies to exhibit long lasting behavioral indices of DPNP (see [22]) references there in). To confirm that the rats used in the present study show behavioral signs of DPNP, we assessed pain behaviors of mechanical and heat hypersensitivity in all rats used $(n=6)$ as we described previously [22]. Briefly, left paw withdrawal latency and left paw withdrawal threshold were assessed in each STZ rat using respectively Hargreaves analgesiometer (Ugo Basile, Comerio, Italy) and an automated von Frey type system known as a dynamic plantar aesthesiometer touch stimulator (Ugo Basile, Italy). Heat hypersensitivity was indicated by a significant decrease in the mean paw withdrawal latency to a noxious heat, whereas mechanical hypersensitivity was indicated by a significant decrease in the mean paw withdrawal threshold.

\subsection{Immunohistochemical experiments}

The immunofluorescent experiments were conducted as we have described previously [24,25]. In brief, control and STZ rats received a lethal dose of the general anesthetic sodium pentobarbital $(80 \mathrm{mg} / \mathrm{kg}$, i.p) and perfused transcardially with phosphate-buff ;ered saline (PBS) (pH 7.4), followed by Lana's fixative (4\% paraformaldehyde (PFA) in $0.1 \mathrm{M}$ PBS, $\mathrm{pH}$ 6.9). This fixative was used based on the findings of pilot experiments that were aimed at testing the following different methods of tissue preparation: (1) no fixation prior to staining (i.e. fresh frozen tissue), (2) a 1:1 methanol/acetone fixative, and (3) a 4\% PFA. Tissue imaging revealed that the cellular architecture of fresh frozen tissue samples was compromised, and that the methanol/acetone fixative caused dehydration and destruction of the neuronal cell membrane, which is not desirable for detecting membrane channel proteins. Thus, the 4\% PFA fixative that is known to preserve cell architecture by crosslinking free amino group, was used. Cross-linking fixatives can also cause the tissue to become more rigid, therefore increasing the strength of delicate tissues whilst preserving the integrity of the cell membrane.

L4/L5 DRGs were extracted from both control and STZ rats that underwent prior behavioral testing to confirm that they exhibit behavioral indices of DPNP as reported previously [22]. The DRG tissue was kept in $10 \%$ sucrose overnight after it had been post fixed for a total of 1-h. The next day it was rapidly snap-frozen in a mixture of isopentane (Fisher-Scientific, UK) and liquid nitrogen (BOC, UK). DRGs from these two groups of rats were serially sectioned at $10 \mu \mathrm{m}$ using a cryostat (Leica CM1950, UK). The DRG tissue was cold mounted onto pre-coated glass slides. To minimize the variability of immunofluorescence, DRG sections from control and STZ rats were mounted onto different halves of the same slide. This is to ensure that both tissues received the same treatment. Omission of the primary antibody was carried out with each round of experiments as a no primary negative control. Immunostaining of tissue was conducted for: (a) DAPI (4', 6-diamidino-2-phenylindole) to visualize cell nuclei, (b) $\mathrm{K}_{\mathrm{v}} 7.2$ and $\mathrm{K}_{\mathrm{v}} 7.5$ channel subunits and (c) Isolectin-B4 (IB4) (1:1000; Sigma-Aldrich, UK).

\subsubsection{Primary antibody characterization}

To select the most appropriate anti- $\mathrm{K}_{\mathrm{v}} 7$ antibodies, pilot experiments of primary antibody screening were carried out. Overall, 13 primary antibodies directed against $\mathrm{K}_{\mathrm{v}} 7.2-\mathrm{K}_{\mathrm{v}} 7.5$ were tested for specificity and staining quality. The primary antibodies deemed to give the best stain were also used for Western blot analysis, to further investigate their specificity. Lysates were run on a $10 \%$ acrylamide gel at $160 \mathrm{~V}$ for $1 \mathrm{~h}$. After staining for $\mathrm{K}_{\mathrm{v}} 7$ proteins, blots were then stripped and reprobed for the 'housekeeping' protein actin to ensure adequate protein transfer had occurred. The primary ion channel antibodies used in this study were: rabbit-anti-Kv7.2 (1:1000, Abcam, Cambridge, MA), and rabbit-anti-Kv7.5 (1:500, Abcam). These antibodies have been used and characterized in other studies (e.g. [20,26]).

\subsubsection{Use of IB4}

IB4 is a neuronal marker for a subpopulation of neurons with unmyelinated C-type fibers $[27,28]$. Together with cell size, IB4 can be used to differentiate between peptidergic (IB4-negative) and non-peptidergic (IB4-positive) DRG nociceptive neurons [29]. Therefore, to determine whether $\mathrm{K}_{\mathrm{v}} 7.2$ and $\mathrm{K}_{\mathrm{v}} 7.3$ subunits are expressed in nonpeptidergic neurons, we examined whether these channels are co-localized with IB4. The plant IB4 lectin (from Griffonia simplicifolia), binds to $\alpha$-D-galactose expressed on cell membranes of a subset of small DRG neurons (Silverman \& Kruger, 1990).

\subsubsection{Image processing and analysis}

Immunofluorescent images were taken at x20 objective 

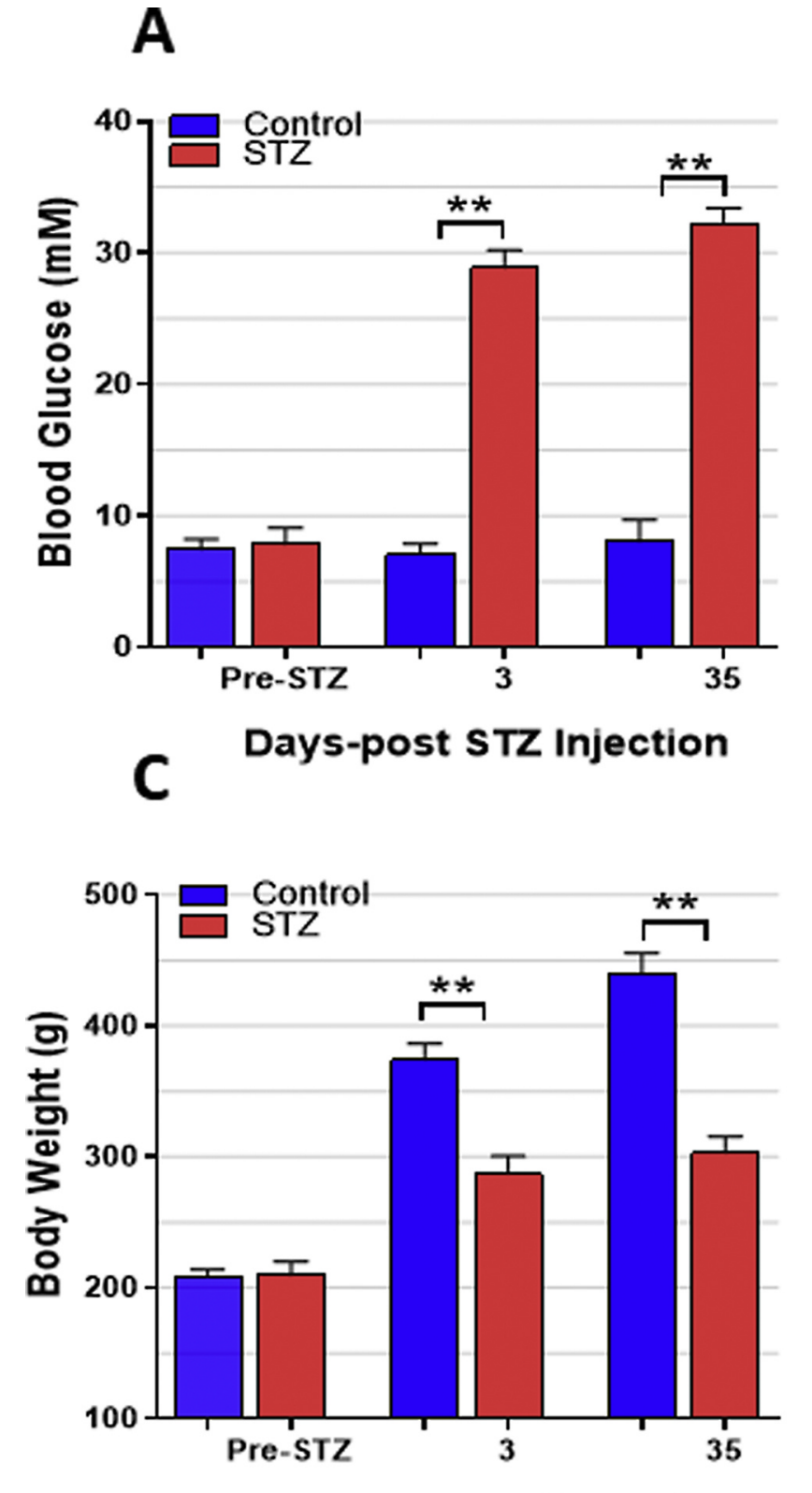

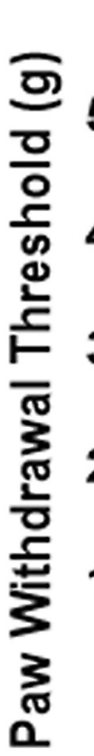

B

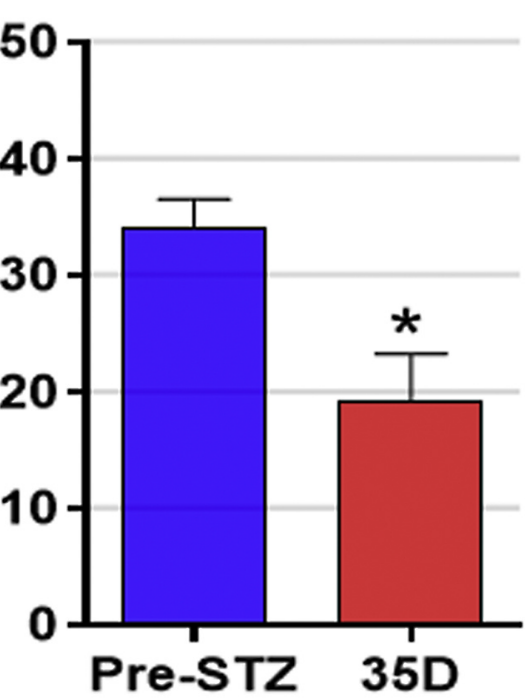

D

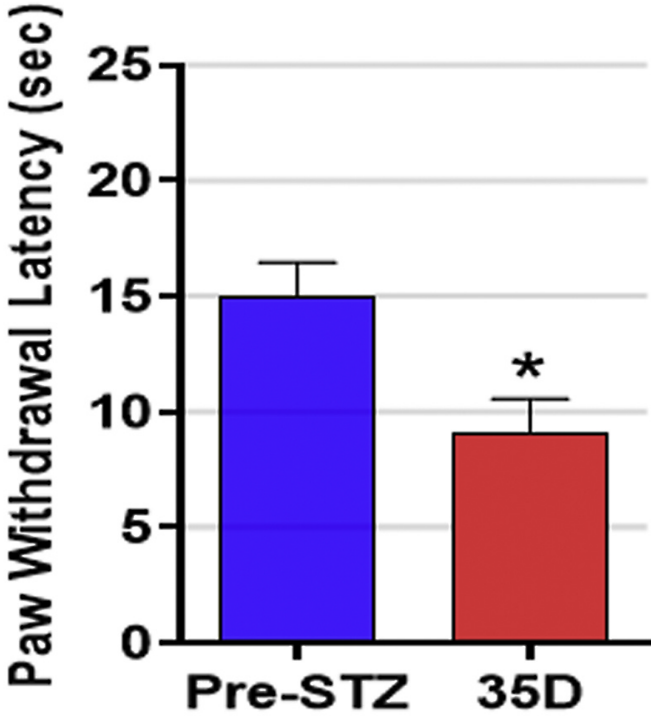

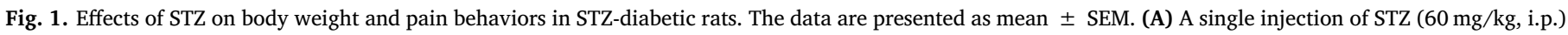

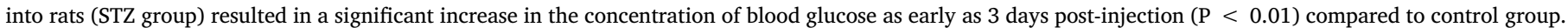

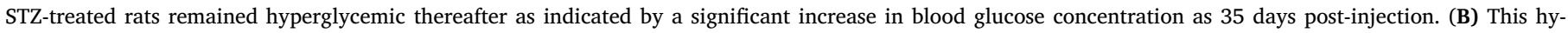

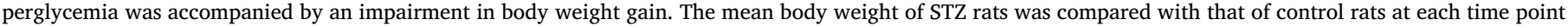

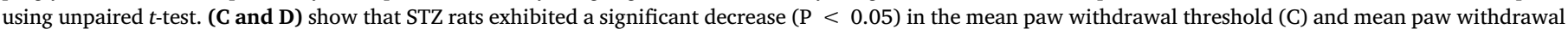

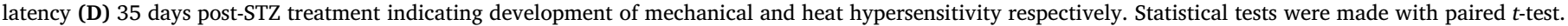
The level of significance is indicated with asterisks as follows: ${ }^{*} \mathrm{P}<0.05$; ${ }^{*} \mathrm{P}<0.01$.

magnification using Axiovision v4.7 software (Carl Zeiss, Germany) with a monochrome camera attached (AxioCam MRm, Carl Zeiss). Densitometry was performed as described previously [24,25].The somatic sizes of $\mathrm{K}_{\mathrm{v}} 7.2$ and $\mathrm{K}_{\mathrm{v}} 7.5$ positive neurons were measured and divided into 3 groups: small-diameter $(<30 \mu \mathrm{m})$ neurons, mediumdiameter (approximately $31-40 \mu \mathrm{m}$ ) neurons, and large-diameter ( $>40 \mu \mathrm{m}$ ) neurons as described previously [24,25]. The staining intensities were plotted against the cell's diameter $(\mu \mathrm{m})$ in a scatter plot. between pre-STZ and post-STZ values were made with $t$-test. The data shown in Fig. 3 and Fig. 4 were compared using the nonparametric Mann-Whitney $U$ test. Spearman's rank correlation coefficient was also used for the data shown in Figs. 3 and 4G. The statistical tests were performed using Graphpad Prism 8 (Graphpad Software, CA, USA). Statistical significance is indicated on graphs with asterisks $(\stackrel{*}{*}<0.05$ and $* P<0.01)$.

\subsection{Statistical analysis}

The data are displayed as mean \pm SEM. Comparisons in Fig. 1 
A
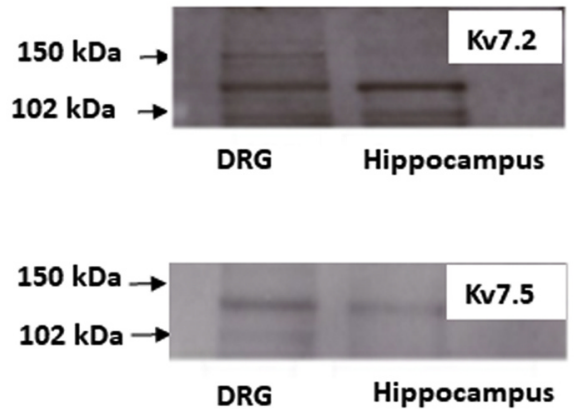

C

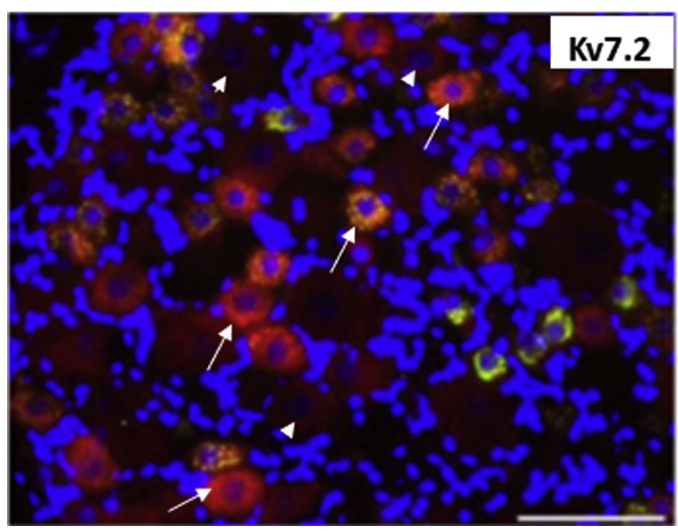

B

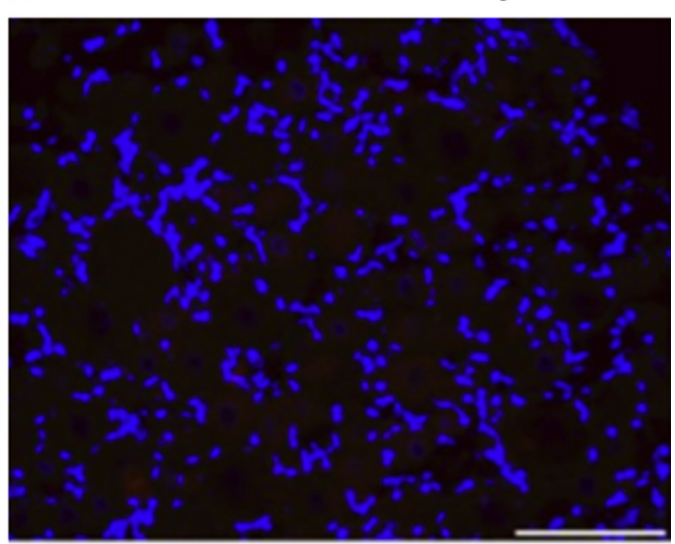

D
Fig. 2. Characterization of anti-Kv7.2 and Kv7.5 channel antibodies using Western Blotting, and expression of Kv7.2 and Kv7.5 channels in DRG neurons. (A) Western blots conducted on rat DRG and hippocampal lysates. Binding of anti-Kv7.2 and Kv7.5 after $20 \mathrm{~min}$ exposure. (B) DAPI staining without application of primary antibodies to stain neuronal nuclei and hence show the presence of cells. (C and D) DAPI staining (blue) with application of anti-Kv7.2 and anti-Kv7.5 antibodies (red) and IB4 stain (green) or yellow (double staining). Note that Kv7.2 and Kv7.5 channel protein is expressed mainly in small DRG neurons both IB4 positive (yellow) and IB4 negative (green) neurons. The arrows in $\mathrm{C}$ and $\mathrm{D}$ indicate the positive neurons, whereas the arrowheads indicate negative or weakly stained neurons. The images were taken at x200 magnification, but for quantification, neurons were routinely observed at $\mathrm{x} 400$. However, even with the lower magnification (x200), some neuronal nuclei could still be seen (large with pale DAPI staining, arrowheads, in C) compared with glial cell nuclei that are smaller, ovoid and more intensely stained by DAPI. Scale bars represent $100 \mu \mathrm{m}$.

\section{Results}

\subsection{Validation of the diabetogenic action of STZ in rats}

We have previously shown that STZ rats exhibited a significant increase in blood glucose concentrations at various time points post STZ [22]. To confirm that the STZ rats used in the present immunohistochemical study are also hyperglycemic, we compared the blood glucose concentrations of STZ rats with those of the control group before STZ administration and 3 and 35 days post-STZ. As shown in Fig. 1A and consistent with our previous findings [22], we found that compared to the control group, all the STZ-treated rats $(n=5)$ exhibited a significant increase $(\mathrm{P}<0.01$, paired $t$-test) in blood concentrations at both time points tested. This hyperglycemic state as accompanied by a significant decrease in the mean body weight in STZ rats. Indeed, as shown in Fig. 1B, STZ rats weighed significantly less than control rats at both 3 and 35 days post-STZ.

\subsection{STZ rats show behavioral signs of mechanical and heat hypersensitivity}

To confirm that the rats used in the present study show behavioral signs of DPNP, we assessed pain behaviors in all rats used. As shown in Fig. 1C and D, STZ rats exhibited significant decreases in the mean paw withdrawal threshold (Fig. 1C) and mean paw withdrawal latency (Fig. 1D) at 35 days post-STZ indicating that STZ-treated rats developed mechanical and heat hypersensitivity respectively.

\subsection{Validation of anti-Kv7 channel antibodies using Western blotting}

To characterize the primary antibodies used in this study, Western blotting was carried out on lysates prepared from rat DRG and hippocampal tissue. As shown in Fig. 2A, the anti-Kv7.2 antibody bound a protein of a molecular mass of between 102 and $150 \mathrm{kDa}$ in rat DRG and hippocampal lysates, which is close to the expected molecular mass of $96 \mathrm{kDa}$. Similarly, the anti-Kv7.5 antibody bound a protein with a molecular mass of between 102 and $150 \mathrm{kDa}$ in rat DRG and hippocampal lysates that is similar to the expected molecular mass of $102 \mathrm{kDa}$ (Fig. 2A). Fig. 2 B shows DAPI staining without application of primary antibodies to stain neuronal nuclei and hence show the presence of cells. As noted in the Methods, double immunofluorescent staining with IB4 was also carried out to determine whether Kv7.2 and Kv7.5 channels are expressed in peptidergic (IB4-negative) or non-peptidergic (IB4-positive) neurons. As shown in Fig. $2 \mathrm{C}$ and D, Kv7.2 and Kv7.5 channel protein is expressed mainly in small DRG IB4 negative neurons.

\subsection{Expression of Kv7.2 and Kv7.5 channels in DRG neurons from control and STZ rats}

We performed immunohistochemistry on rat L4/L5 DRGs from control and STZ rats, using anti-Kv7.2 and Kv7.5 antibodies to investigate whether expression of these subunits changes in STZ-diabetic rats 35 days post STZ. We focused on these subunits because these isoforms are clearly expressed in rodent DRG neurons (see Introduction) and because we do not have reliable and selective antibodies for the other neuronal Kv7.4 and Kv7.3 subunits.

\subsubsection{Expression of Kv7.2 and Kv7.5 channels in control DRG neurons}

We found that in control DRGs, Kv7.5- immunoreactivity (IR) was seen mainly in small DRG neurons. As shown in Fig. 3C double immunofluorescent staining show that all IB4-positive neurons showed Kv7.5-IR. However, weak Kv7.5-IR is also seen in medium/large sized neurons (Fig. 3C). Similar to Kv7.5-IR, Kv7.2 is also expressed mainly in small neurons (both IB4-positive and IB4-negative) (Fig. 4 C). Weak Kv7.2 staining is also seen in the cytoplasm of medium and large-sized 


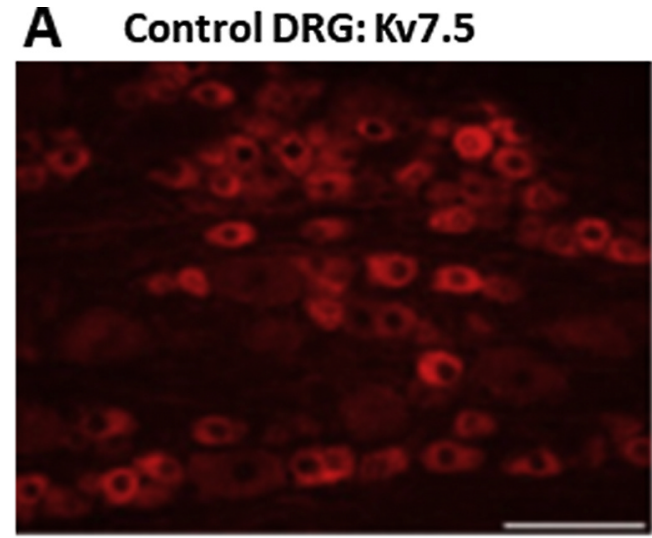

B

IB4

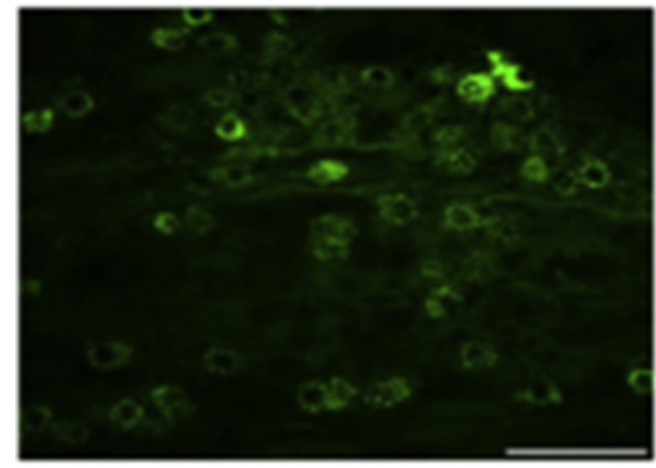

C Control: Overlay

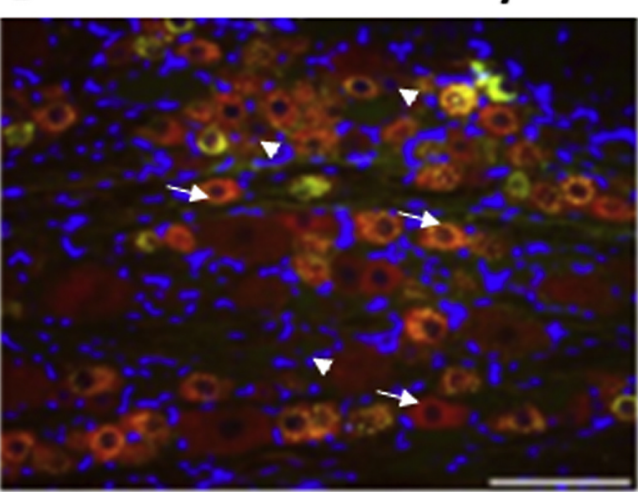

D

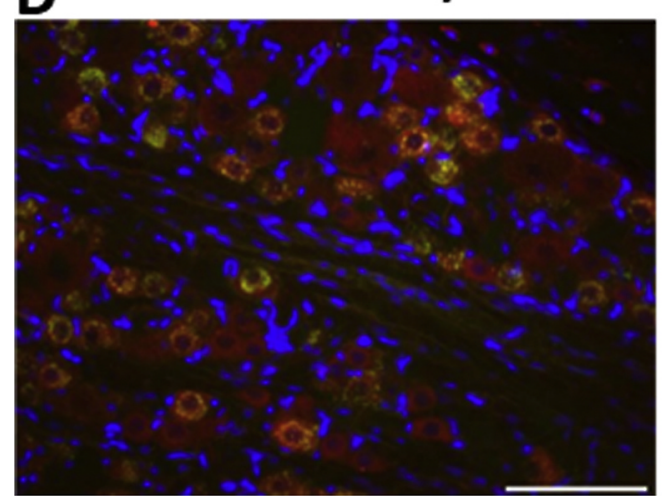

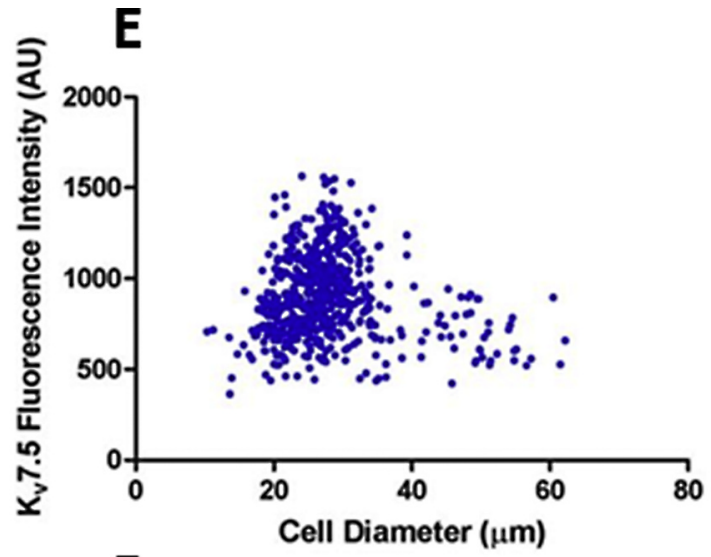

$\mathbf{F}$
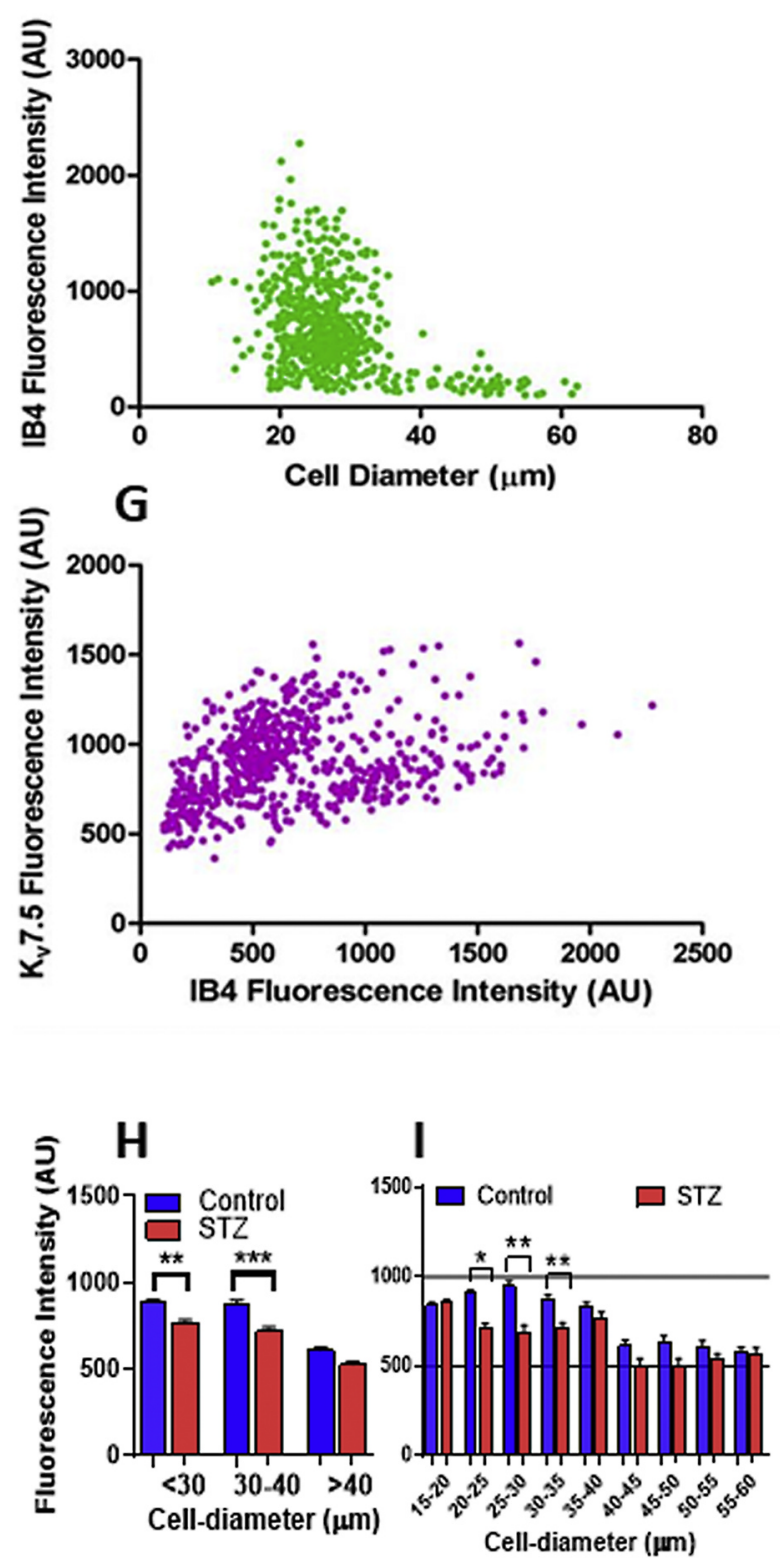


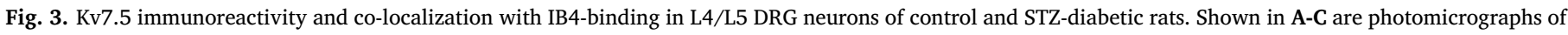

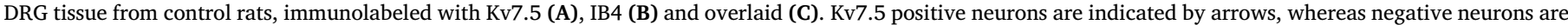

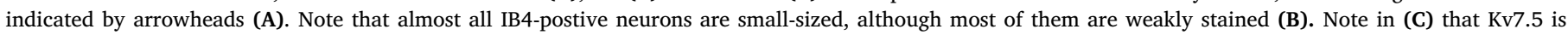

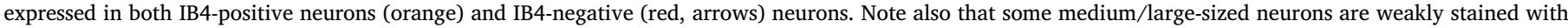

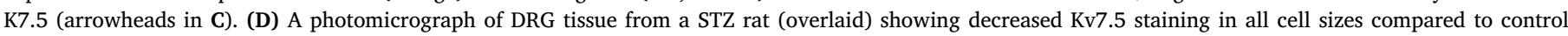

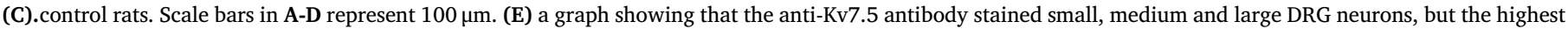

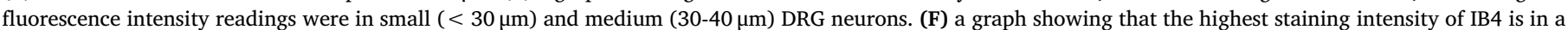

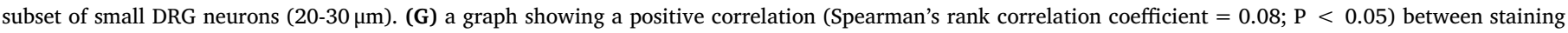

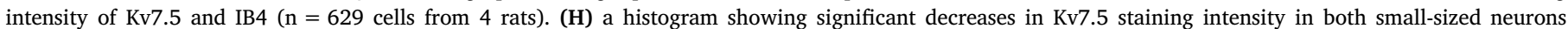

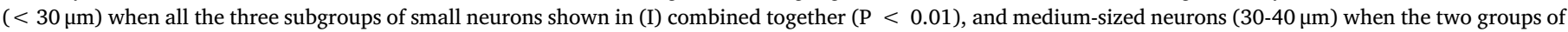

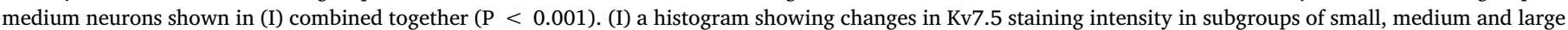

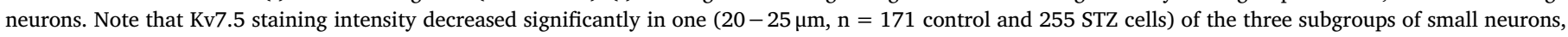

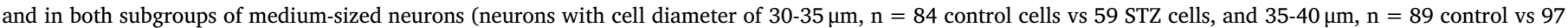

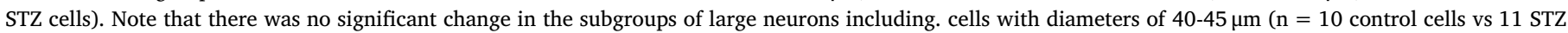
cells) and $45-50 \mu \mathrm{m}(\mathrm{n}=17$ control cells vs $8 \mathrm{STZ}$ cells). The data in $\mathrm{H}$ and I are presented as mean \pm SEM and were analyzed using a Mann-Whitney U test.

neurons (Fig. 4C). Double immunofluorescent staining data revealed that all IB4-postive neurons were Kv7.2-postive (Fig. 4B and C). However, as shown in Fig. 4C many IB4-negative neurons were Kv7.2postive, suggesting some functional significance of this isoform in both peptidergic and non-peptidergic nociceptors. As shown in Figs. 3 and 4, staining of both $\mathrm{K}_{\mathrm{V}} 7.2$ and Kv7.5 in small DRG neurons was mainly cytoplasmic. The weak staining of Kv7.2 in medium- and large-sized neurons was also cytoplasmic.

\subsubsection{STZ induces down regulation in Kv7.5 channel expression, and} increased Kv7.2 expression in DRG neurons

We found 35 days post STZ, a significant decrease in $\mathrm{K}_{\mathrm{v}} 7.5$-IR in small- $(<30 \mu \mathrm{m})$ and medium- $(30-40 \mu \mathrm{m})$ sized DRG neurons, determined by densitometric comparison of DRG sections from control and STZ rats (Fig. $3 \mathrm{H}$ and I). Fig. $3 \mathrm{H}$ shows significant decreases (Mann-Whitney $U$ test) in $\mathrm{K}_{\mathrm{v}}$ 7.5-IR in small neurons $(\mathrm{P}<0.01$ ) when the three subgroups of small neurons $(<30 \mu \mathrm{m})$ shown in Fig. 3 I were combined together. There was also a significant decrease in $\mathrm{K}_{\mathrm{v}} 7.5$-IR in medium-sized neurons $(\mathrm{P}<0.001)$ when the two subgroups of medium DRG neurons $(30-40 \mu \mathrm{m})$ were combined together (Fig. $3 \mathrm{H}$ ). Fig. 3I shows significant decreases in $\mathrm{K}_{\mathrm{v}}$ 7.5-IR in subgroups of small and medium-sized DRG neurons. Actually, the decrease in $\mathrm{K}_{\mathrm{v}} 7.5$-IR was even more statistically significant $(P<0.001)$ when the subgroups of medium-sized neurons (neurons with cell diameter of $30-35 \mu \mathrm{m}$ and neurons with cell diameter of $35-40 \mu \mathrm{m}$ ) were combined together (Fig. 3H).

Unexpectedly and in stark contrast to Kv7.5-IR, the intensity of $\mathrm{K}_{\mathrm{v}} 7.2$-IR increased in all cell sizes particularly in IB4-negative neurons (Fig. 4D). Indeed as shown in Fig. 4H, there was an apparent increase in Kv7.2 staining intensity in all cell sizes of DRG neurons, but such increase in Kv7.2-IR was significant $(\mathrm{P}<0.001)$ only in small-sized $(<30 \mu \mathrm{m})$ neurons when the three subgroups of small neurons $(<30 \mu \mathrm{m})$ were combined together (Fig. $4 \mathrm{H})$. Fig. $4 \mathrm{I}$ shows that the subgroup of small DRG neurons that showed significant increase in $\mathrm{K}_{\mathrm{v}}$ 7.2-IR was that with cell diameter of $20-25 \mu \mathrm{m}(\mathrm{P}<0.01$, Mann-Whitney $U$ test).

\section{Discussion}

In the present immunohistochemical study, we have investigated, in the STZ model of DPNP, whether expression of $\mathrm{K}_{\mathrm{v}} 7.2$ and/or $\mathrm{K}_{\mathrm{v}} 7.5$ channel protein is altered in DRG neurons. Our findings show, 35 days post-STZ, a significant decrease in $\mathrm{K}_{\mathrm{v}} 7.5$ channel expression in small $(<30 \mu \mathrm{m})$ and medium $(30-40 \mu \mathrm{m})$ sized neurons, and increased expression of $\mathrm{K}_{\mathrm{v}} 7.2$ channel protein in all sizes of DRG neurons particularly in IB4 negative small neurons $(<30 \mu \mathrm{m})$. The decrease in $\mathrm{K}_{\mathrm{v}} 7.5$ channel expression in small and medium-sized DRG neurons is likely to contribute to the mechanisms of their hyperexcitability and the resulting pain hypersensitivity associated with DPNP. Such decrease in $\mathrm{K}_{\mathrm{v}} 7.5$ channel expression may be due to a dramatic decrease in expression of this subunit in the subpopulation of DRG neurons to a degree under the detection threshold. The upregulation of $\mathrm{K}_{\mathrm{v}} 7.2$ subunit may be an activity dependent compensatory mechanism to limit STZ-induced hyperexcitability of DRG neurons and the associated pain hypersensitivity.

The rat STZ model that we have used in the present study is a wellestablished model of DPNP used by numerous studies investigating the pathophysiology of DPNP. We and others have previously shown this model to exhibit long lasting behavioral signs of DPNP including mechanical and heat hypersensitivity, but not cold hypersensitivity or spontaneous pain behavior (see [22] and references therein). Our current behavioral findings are consistent with our previous studies [22].The mechanical hypersensitivity behavior is believed to correspond to the cutaneous hypersensitivity in patients with diabetic neuropathy that leads to evoked pain on contact with an external stimulus, such as clothing, and that can be so painful in some patients preventing them from performing their daily activities (see [22] and references therein).

Our findings of downregulation of $\mathrm{K}_{\mathrm{v}} 7.5$ channel protein expression in small DRG neurons are partly consistent with those of [20] who also found significant decreases in the expression of $\mathrm{K}_{\mathrm{v}} 7.2, \mathrm{~K}_{\mathrm{v}} 7.3$ and $\mathrm{K}_{\mathrm{v}} 7.5$ channels at both mRNA and protein levels in STZ rats. $\mathrm{K}_{\mathrm{v}} 7.5$ expression was also found to be down-regulated in DRG neurons following sciatic nerve axotomy [17]. Taken together, these findings suggest that downregulation of $\mathrm{K}_{\mathrm{v}} 7.5$ channels might be a common mechanism for pain hypersensitivity in different types of PNP. However our findings are not in line with the findings of these studies [20] and other previous studies who found a down-regulation in $\mathrm{K}_{\mathrm{v}} 7.2$ expression in DRG neurons in other models of PNP including partial sciatic nerve ligation [16], axotomy [30], rat models of bone cancer pain [19] and chemotherapy-induced PNP [31]. The down-regulation of $\mathrm{K}_{\mathrm{v}} 7.2$ expression induced by the partial sciatic nerve ligation in rat DRG neurons is dependent on repressor element 1-silencing transcription factor [16]. Such down regulation in $\mathrm{K}_{\mathrm{v}} 7.2$ expression is believed to contribute to hyperexcitability of DRG neurons and the resulting pain hypersensitivity associated with PNP in these animal models [32].

Our findings of up-regulation of $\mathrm{K}_{\mathrm{v}} 7.2$ channels in small DRG neurons in STZ rats that is likely to result in decreased excitability of these DRG neurons are unexpected. However, this subunit was also found to be upregulated in primary afferent trigeminal ganglion neurons using the infraorbital nerve chronic constrictive injury model of PNP [26]. Increased expression of $\mathrm{K}_{\mathrm{v}} 7.2$ (and $\mathrm{K}_{\mathrm{v}} 7.3$ ) channels was also found in DRG neurons 7 days post-nerve injury in the SNL model of PNP [33], but such increase was transient and returned to normal levels 19 days after nerve injury [33]. It is noteworthy that expression of $\mathrm{K}_{\mathrm{v}} 7.2 / \mathrm{K}_{\mathrm{v}} 7.3$ channels in hippocampal neurons was found to undergo transcriptional up-regulation in an activity dependent manner in a rodent model of epilepsy [34], a neurological condition that is also characterized by neuronal hyperexcitability. These co-workers reported that such upregulation involves activation of calcineurin and nuclear factor of 

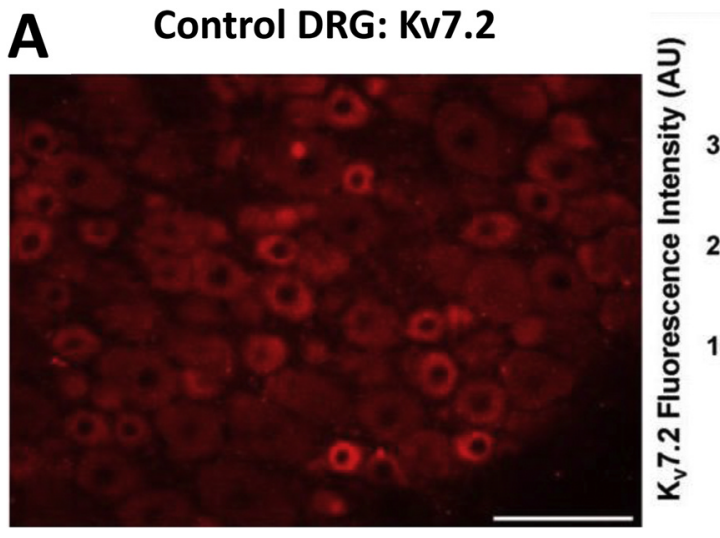

\section{E}

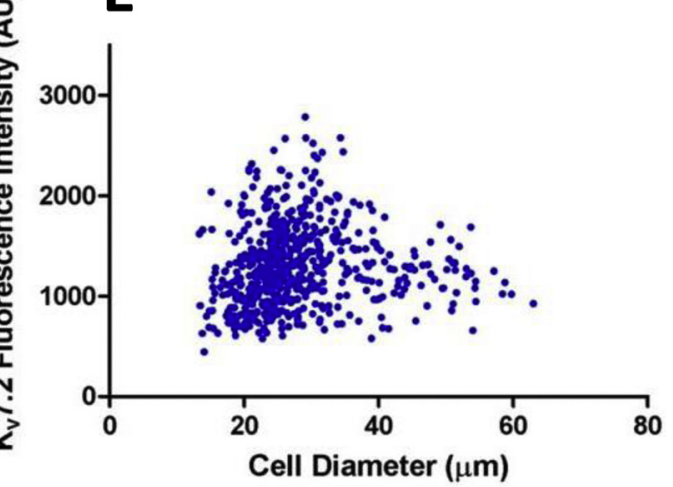

B IB4
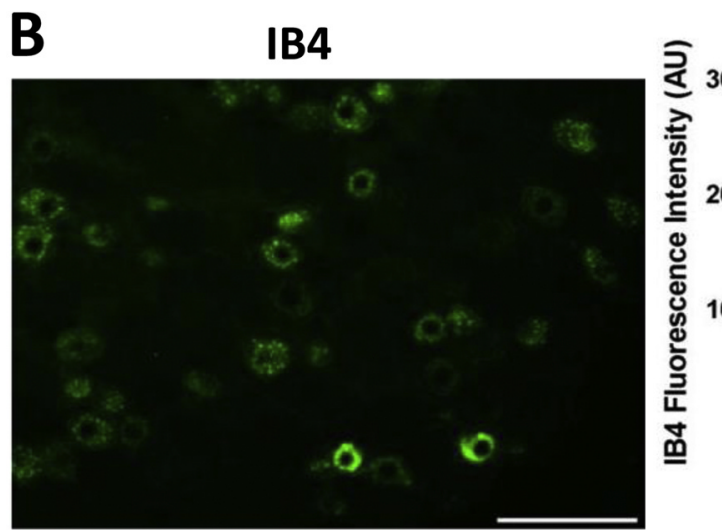

$\mathbf{F}$

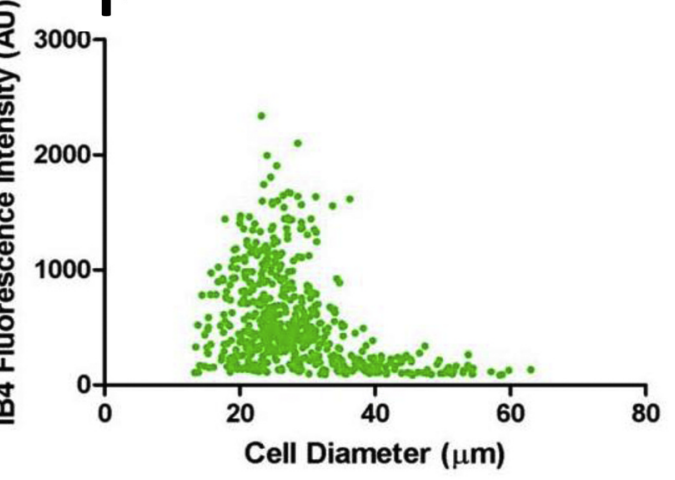

\section{Control: Overlay}
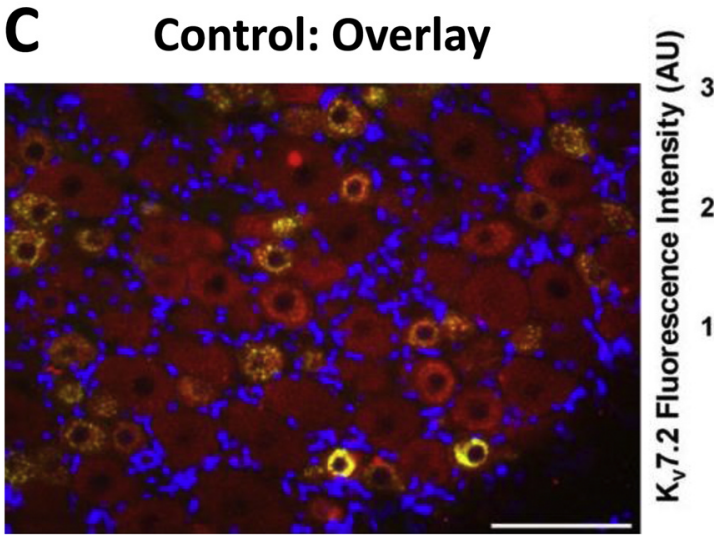

Fig. 4. Kv7.2 immunoreactivity and co-localization with IB4-binding in L4/L5 DRG neurons of control and STZ-diabetic rats. (A-C) are photomicrographs of DRG tissue from control rats, immunolabeled with Kv7.5 (A), IB4 (B) and overlaid (C). Note that Kv7.2 is expressed in both IB4 negative (arrows) and IB4 positive (arrowheads) neurons. (D) is a photomicrograph of DRG tissue from a STZ rat (overlaid) showing increased Kv7.2 staining mainly in medium and large-sized neuronal cells compared to control (C). Details for E-G are as in Fig.3 legend with the exception that there was no significant correlation between Kv7.2 and IB4 staining. (H). a histogram showing a significant increase in Kv7.5 staining intensity in small-sized neurons $(<30 \mu \mathrm{m})$ when all the three subgroups of small neurons shown in (I) were combined together $(\mathrm{P}<0.001)$. Note that there was no significant change in mediumand large sized neurons. (I). a histogram showing changes in Kv7.2 staining intensity in subgroups of small, medium and large neurons. Note that of the different subgroups, Kv7.2 staining intensity increase significantly only in one of the three subgroups of small neuron (neurons with cell diameter of $20-25 \mu \mathrm{m}$ ( $n=185$ control cells vs 243 STZ cells). For further details, see Fig. 3 legend.

\section{STZ: Overlay}
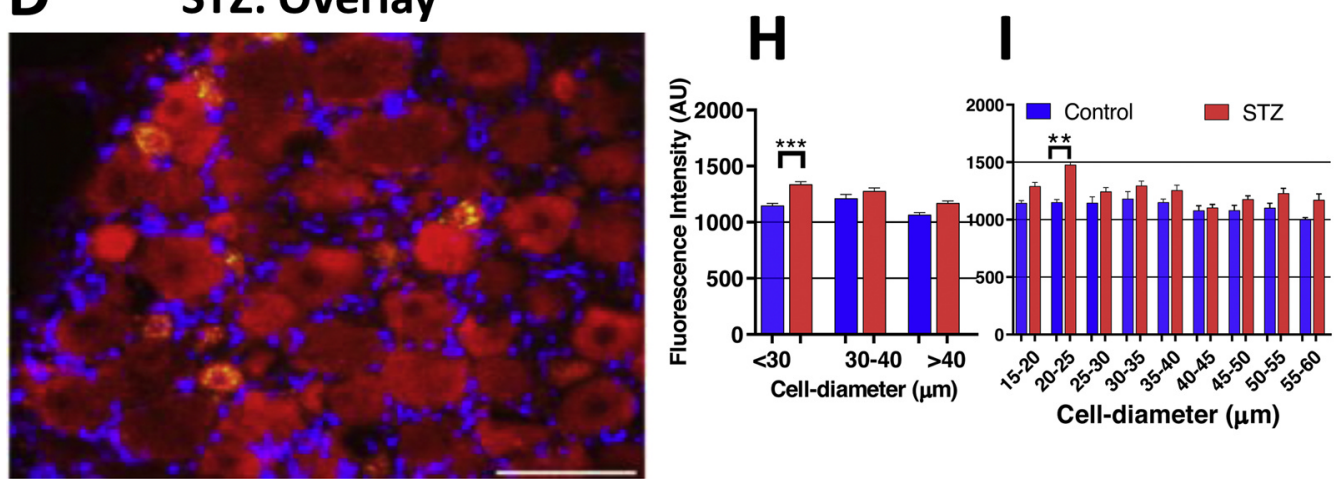

activated T cell (NFAT) transcription factors that requires $\mathrm{Ca}^{2+}$ influx through L-type $\mathrm{Ca}^{2+}$ channels [34]. Thus, it is possible that the upregulation of $\mathrm{K}_{\mathrm{v}} 7.2$ channels, that we found in small DRG neurons in STZ rats, may also be due to an activity dependent transcriptional regulation.

Our findings that $\mathrm{K}_{\mathrm{v}} 7.2$-IR is observed mainly in small-sized DRG neurons (and weakly in some medium/large neurons) in the control group are consistent with those of Passmore et al. [35] who found that 
this subunit to be expressed by both small and large diameter DRG neurons. They are also consistent with those of a previous study [16] showing $\mathrm{K}_{\mathrm{v}} 7.2$ to be the predominant subunit in C-type DRG neurons. However, our findings are in contrast with those of King and his coworkers who reported that $\mathrm{k}_{\mathrm{v}} 7.2$-IR was most prominent in large diameter DRG neurons $[17,36]$ most of which are A $\beta$-low-threshold mechanoreceptive (LTMs) [37]. To determine whether $K_{v} 7.2$ and $K_{v} 7.5$ channels are expressed in peptidergic (IB4-negative) or non-peptidergic (IB4-positive) neurons, we examined the DRG tissue from control and STZ rats for co-localization of $\mathrm{K}_{\mathrm{v}} 7.2$ and $\mathrm{K}_{\mathrm{v}} 7.5$ with the plant isolectin IB4, a neuronal marker for a subpopulation of unmyelinated C-fiber neurons $[27,28])$. We found that all IB4-positive neurons expressed $\mathrm{K}_{\mathrm{v}} 7.2$ and $\mathrm{K}_{\mathrm{v}} 7.5$ subunits suggesting that these channels are involved in regulating excitability of non-peptidergic neurons. However, it should be noted that not all IB4-positive neurons are nociceptors. Indeed, we have previously demonstrated that a small number of LTMs were weakly stained for IB4 [28]. We did not investigate co-localization of Kv7.2 and Kv7.5 subunits in DRG neurons, but previous studies reported that these subunits co-localize in DRG neurons $[19,35]$. However, they do not seem to combine with each other [38,39], but instead they are thought to homotetramerise, or heterotetramerise with Kv7.3 that has been shown to readily combine with other $K_{v} 7$ subunits $[35,38,39]$.

We have not examined expression of the other neuronal $\mathrm{K}_{\mathrm{v}} 7$ channels ( $\mathrm{K}_{\mathrm{v}} 7.3$ and $\mathrm{K}_{\mathrm{v}} 7.4$ subunits) in DRG neurons because as already noted we did not have reliable and selective antibodies for these subunits. However, $\mathrm{K}_{\mathrm{v}} 7.3$ has been shown to be co-localized with $\mathrm{K}_{\mathrm{v}} 7.2$ in DRGs [40-42], but it is believed not to be the major $\mathrm{K}_{\mathrm{v}} 7$ subtype in nociceptors $[17,36]$. Consistent with these latter findings, $\mathrm{K}_{\mathrm{v}} 7.3$ was found to be co-localized in DRG somata with NF200, a marker for large neurons with myelinated fibers (putative LTM neurons) [43], and TrkB, a general marker of A $\delta$-LTMs [42]. $\mathrm{K}_{\mathrm{v}} 7.4$ seems to be detected mainly in a small subset of rapidly adapting LTM DRG neurons [44], but neither $\mathrm{K}_{\mathrm{v}} 7.4$ nor Kv7.5 were found in A $\delta$-LTMs [42]

In conclusion our findings of decreased $\mathrm{K}_{\mathrm{v}} 7.5$ channel expression in small and medium sized DRG neurons (putative nociceptors) suggest that the subunit is likely to contribute to the hyperexcitability of this subpopulation of DRG neurons and the resulting pain hypersensitivity in STZ rats. On the other hand, the increased expression of Kv7.2 channels that has also been reported in other models of chronic pain might be a compensatory mechanism to limit STZ-induced hyperexcitability of DRG neurons and the associated pain hypersensitivity.

\section{Funding}

This research work was supported by a Medical Research Council grant (G0700420) and a PhD studentship from Biotechnology and Biological Sciences Research Council to L. Djouhri.

\section{CRediT authorship contribution statement}

Laiche Djouhri: Conceptualization, Supervision, Data curation, Writing - original draft, Writing - review \& editing. Asad Zeidan: Data curation, Writing - review \& editing. Seham A. Abd El-Aleem: Writing - review \& editing.

\section{Declaration of Competing Interest} clare.

The authors have no potential or actual conflicts of interest to de-

\section{Acknowledgements}

We thank staff at BSU of University of Liverpool for their technical support and help.

\section{Appendix A. Supplementary data}

Supplementary material related to this article can be found, in the online version, at doi:https://doi.org/10.1016/j.neulet.2020.135277.

\section{References}

[1] C.A. Lee-Kubli, N.A. Calcutt, Painful neuropathy: mechanisms, Handb. Clin. Neurol. 126 (2014) 533-557.

[2] C.A. Abbott, R.A. Malik, E. R. van Ross, J. Kulkarni, A.J. Boulton, Prevalence and characteristics of painful diabetic neuropathy in a large community-based diabetic population in the U.K, Diabetes Care 34 (2011) 2220-2224.

[3] D. Bouhassira, M. Letanoux, A. Hartemann, Chronic pain with neuropathic characteristics in diabetic patients: a French cross-sectional study, PLoS One 8 (2013) e74195.

[4] A.K. Schreiber, C.F. Nones, R.C. Reis, J.G. Chichorro, J.M. Cunha, Diabetic neuropathic pain: physiopathology and treatment, World J. Diabetes 6 (2015) 432-444.

[5] S.J. Benbow, A.W. Chan, D. Bowsher, I.A. MacFarlane, G. Williams, A prospective study of painful symptoms, small-fibre function and peripheral vascular disease in chronic painful diabetic neuropathy, Diabet. Med. 11 (1994) 17-21.

[6] H. Kim, J.J. Kim, Y.S. Yoon, Emerging therapy for diabetic neuropathy: cell therapy targeting vessels and nerves, Endocr. Metab. Immune Disord. Drug Targets 12 (2012) 168-178.

[7] R. Baron, A. Binder, G. Wasner, Neuropathic pain: diagnosis, pathophysiological mechanisms, and treatment, Lancet Neurol. 9 (2010) 807-819.

[8] K.J. Burchiel, L.C. Russell, R.P. Lee, A.A. Sima, Spontaneous activity of primary afferent neurons in diabetic BB/Wistar rats. A possible mechanism of chronic diabetic neuropathic pain, Diabetes 34 (1985) 1210-1213.

[9] S.C. Ahlgren, J.F. Wang, J.D. Levine, C-fiber mechanical stimulus-response functions are different in inflammatory versus neuropathic hyperalgesia in the rat, Neuroscience 76 (1997) 285-290.

[10] X. Chen, J.D. Levine, Hyper-responsivity in a subset of C-fiber nociceptors in a model of painful diabetic neuropathy in the rat, Neuroscience 102 (2001) 185-192.

[11] G.M. Khan, S.R. Chen, H.L. Pan, Role of primary afferent nerves in allodynia caused by diabetic neuropathy in rats, Neuroscience 114 (2002) 291-299.

[12] T.J. Jentsch, Neuronal KCNQ potassium channels: physiology and role in disease, Nat. Rev. Neurosci. 1 (2000) 21-30.

[13] P. Delmas, D.A. Brown, Pathways modulating neural KCNQ/M (Kv7) potassium channels, Nat. Rev. Neurosci. 6 (2005) 850-862.

[14] D.A. Brown, G.M. Passmore, Neural KCNQ (Kv7) channels, Br. J. Pharmacol. 156 (2009) 1185-1195.

[15] A.D. Wickenden, G. McNaughton-Smith, Kv7 channels as targets for the treatment of pain, Curr. Pharm. Des. 15 (2009) 1773-1798.

[16] K. Rose, L. Ooi, C. Dalle, B. Robertson, I.C. Wood, N. Gamper, Transcriptional repression of the M channel subunit Kv7.2 in chronic nerve injury, Pain 152 (2011) 742-754.

[17] C.H. King, S.S. Scherer, Kv7.5 is the primary Kv7 subunit expressed in C-fibers, J. Comp. Neurol. 520 (2012) 1940-1950.

[18] K.Z. Duan, Q. Xu, X.M. Zhang, Z.Q. Zhao, Y.A. Mei, Y.Q. Zhang, Targeting A-type K $(+)$ channels in primary sensory neurons for bone cancer pain in a rat model, Pain 153 (2012) 562-574.

[19] O. Zheng, D. Fang, M. Liu, J. Cai, Y. Wan, J.S. Han, G.G. Xing, Suppression of KCNQ/M (Kv7) potassium channels in dorsal root ganglion neurons contributes to the development of bone cancer pain in a rat model, Pain 154 (2013) 434-448.

[20] T. Yu, L. Li, H. Liu, H. Li, Z. Liu, Z. Li, KCNQ2/3/5 channels in dorsal root ganglion neurons can be therapeutic targets of neuropathic pain in diabetic rats, Mol. Pain 14 (2018) 1744806918793229.

[21] L. Djouhri, A. Zeidan, S.A. Abd El-Aleem, T. Smith, Cutaneous abeta-non-nociceptive, but not C-nociceptive, dorsal root ganglion neurons exhibit spontaneous activity in the streptozotocin rat model of painful diabetic neuropathy in vivo, Front. Neurosci. 14 (2020) 530.

[22] L. Djouhri, M.I. Malki, A. Zeidan, K. Nagi, T. Smith, Activation of Kv7 channels with the anticonvulsant retigabine alleviates neuropathic pain behaviour in the streptozotocin rat model of diabetic neuropathy, J. Drug Target. 27 (2019) 1118-1126.

[23] R.M. Deacon, Housing, husbandry and handling of rodents for behavioral experiments, Nat. Protoc. 1 (2006) 936-946.

[24] X. Weng, T. Smith, J. Sathish, L. Djouhri, Chronic inflammatory pain is associated with increased excitability and hyperpolarization-activated current (Ih) in C- but not Adelta-nociceptors, Pain 153 (2012) 900-914.

[25] T. Smith, M. Al Otaibi, J. Sathish, L. Djouhri, Increased expression of HCN2 channel protein in L4 dorsal root ganglion neurons following axotomy of L5- and inflammation of L4-spinal nerves in rats, Neuroscience 295 (2015) 90-102.

[26] J. Ling, F. Erol, J.G. Gu, Role of KCNQ2 channels in orofacial cold sensitivity: KCNQ2 upregulation in trigeminal ganglion neurons after infraorbital nerve chronic constrictive injury, Neurosci. Lett. 664 (2018) 84-90.

[27] P.D. Kitchener, P. Wilson, P.J. Snow, Selective labelling of primary sensory afferent terminals in lamina II of the dorsal horn by injection of Bandeiraea simplicifolia isolectin B4 into peripheral nerves, Neuroscience 54 (1993) 545-551.

[28] X. Fang, L. Djouhri, S. McMullan, C. Berry, S.G. Waxman, K. Okuse, S.N. Lawson, Intense isolectin-B4 binding in rat dorsal root ganglion neurons distinguishes $\mathrm{C}$ fiber nociceptors with broad action potentials and high Nav1.9 expression, J. Neurosci. 26 (2006) 7281-7292.

[29] C.L. Stucky, G.R. Lewin, Isolectin B(4)-positive and -negative nociceptors are functionally distinct, J. Neurosci. 19 (1999) 6497-6505. 
[30] E. Cisneros, C. Roza, N. Jackson, J.A. Lopez-Garcia, A new regulatory mechanism for Kv7.2 protein during neuropathy: enhanced transport from the soma to axonal terminals of injured sensory neurons, Front. Cell. Neurosci. 9 (2015) 470.

[31] J. Ling, F. Erol, V. Viatchenko-Karpinski, H. Kanda, J.G. Gu, Orofacial neuropathic pain induced by oxaliplatin: downregulation of KCNQ2 channels in V2 trigeminal ganglion neurons and treatment by the KCNQ2 channel potentiator retigabine, Mol. Pain 13 (2017) 1744806917724715.

[32] X. Du, H. Gao, D. Jaffe, H. Zhang, N. Gamper, M-type K(+) channels in peripheral nociceptive pathways, $\mathrm{Br}$. J. Pharmacol. 175 (2018) 2158-2172.

[33] A. Wickenden, $\mathrm{K}(+)$ channels as therapeutic drug targets, Pharmacol. Ther. 94 (2002) 157-182

[34] J. Zhang, M.S. Shapiro, Activity-dependent transcriptional regulation of M-Type (Kv7) K(+) channels by AKAP79/150-mediated NFAT actions, Neuron 76 (2012) 1133-1146.

[35] G.M. Passmore, A.A. Selyanko, M. Mistry, M. Al-Qatari, S.J. Marsh, E.A. Matthews, A.H. Dickenson, T.A. Brown, S.A. Burbidge, M. Main, D.A. Brown, KCNQ/M currents in sensory neurons: significance for pain therapy, J. Neurosci. 23 (2003) $7227-7236$

[36] C.H. King, E. Lancaster, D. Salomon, E. Peles, S.S. Scherer, Kv7.2 regulates the function of peripheral sensory neurons, J. Comp. Neurol. 522 (2014) 3262-3280.

[37] L. Djouhri, S.N. Lawson, Abeta-fiber nociceptive primary afferent neurons: a review of incidence and properties in relation to other afferent A-fiber neurons in mammals, Brain Res. Brain Res. Rev. 46 (2004) 131-145.

[38] C. Lerche, C.R. Scherer, G. Seebohm, C. Derst, A.D. Wei, A.E. Busch, K. Steinmeyer,
Molecular cloning and functional expression of KCNQ5, a potassium channel subunit that may contribute to neuronal M-current diversity, J. Biol. Chem. 275 (2000) 22395-22400.

[39] B.C. Schroeder, M. Hechenberger, F. Weinreich, C. Kubisch, T.J. Jentsch, KCNQ5, a novel potassium channel broadly expressed in brain, mediates M-type currents, J. Biol. Chem. 275 (2000) 24089-24095.

[40] Z. Pan, T. Kao, Z. Horvath, J. Lemos, J.Y. Sul, S.D. Cranstoun, V. Bennett, S.S. Scherer, E.C. Cooper, A common ankyrin-G-based mechanism retains KCNQ and NaV channels at electrically active domains of the axon, J. Neurosci. 26 (2006) 2599-2613.

[41] E.C. Cooper, Made for "anchorin": Kv7.2/7.3 (KCNQ2/KCNQ3) channels and the modulation of neuronal excitability in vertebrate axons, Semin. Cell Dev. Biol. 22 (2011) 185-192.

[42] S. Schutze, I.J. Orozco, T.J. Jentsch, KCNQ potassium channels modulate sensitivity of skin down-hair (D-hair) mechanoreceptors, J. Biol. Chem. 291 (2016) $5566-5575$.

[43] S.N. Lawson, P.J. Waddell, Soma neurofilament immunoreactivity is related to cell size and fibre conduction velocity in rat primary sensory neurons, J. Physiol. 435 (1991) 41-63.

[44] M. Heidenreich, S.G. Lechner, V. Vardanyan, C. Wetzel, C.W. Cremers, E.M. De Leenheer, G. Aranguez, M.A. Moreno-Pelayo, T.J. Jentsch, G.R. Lewin, KCNQ4 K $(+)$ channels tune mechanoreceptors for normal touch sensation in mouse and man, Nat. Neurosci. 15 (2011) $138-145$. 